\title{
The effects of strengthening exercise, stretching and meditation on electromyography onset timing of the rectus femoris and gastrocnemius during vertical jump performance in healthy adults
}

\author{
Ji Young Eum, Yeoung Kyun Kim, Eun Ji Park, Ju Hee Lee, Ji Eun Lee, Jin Ju Lim, Man Ho Choi, Hyun Hee Kim
}

Department of Physical Therapy, Shinsung University, Dangjin, Republic of Korea

Objective: Jump training helps increase the muscle power by improving the muscle strength and reaction time of the muscle in operation. The purpose of this study was to identify the effects of strengthening, stretching exercise and meditation on electromyographic (EMG) onset timing of rectus femoris and gastrocnemius muscle during vertical jump performance.

Design: Cross-sectional study.

Methods: Ten healthy adults ( 5 male and 5 female) who were familiar with the vertical jumping task and had no lower extremity injuries or any bone or joint disorders, were recruited for this study. Muscle onset timing was measured by surface EMG. After EMG onset timing were measured during performing three baseline vertical jump trials, strengthening and stretching exercises of the rectus femoris and gastrocnemius, and meditation were performed in random order. EMG onset timing was measured during vertical jump after intervention, respectively. EMG value was averaged for the three trials and analyzed using one-way repeated ANOVA.

Results: During vertical jump, EMG onset timing of gastrocnemius was a significant difference after intervention ( $p<0.05)$, and then there was significantly faster in strengthening exercise than meditation $(p<0.05)$.

Conclusions: These results indicate the potential positive effect of performing strengthening exercise of the gastrocnemius before a jumping event. Future research is required to identify the effects of intervention over a long period.

Key Words: Meditation, Electromyography, Exercise, Meditation

\section{Introduction}

The muscle activities in human body may be divided into the eccentric contraction, in which the muscle length shortens much further, concentric contraction, in which the muscle length shortens a little, and the isometric contraction, in which there is no change in the muscle length.

The eccentric contraction and concentric contraction play the basic role in various movements such as walking in daily life, running and jumping and changing direction in sport games. When a human is making many movements, the ec- centric contraction precedes the eccentric contraction, and this physical phenomenon is called stretch-shortening cycle [1]. Athletes are trained to make stretch-shortening cycle occur faster and stronger in order to cut their time off or renew their record in various sports, such as jump, running and throwing [2].

The increase in muscle strength of rectus femoris has improved the torque of the muscle [3,4]. Jones and Rutherford [3] reported that strength training the main change in the first 12 weeks is an increase in the force generated per unit cross-sectional area of muscle, and the increase in muscular

Received: 15 May, 2015 Revised: 11 June, 2015 Accepted: 11 June, 2015

Corresponding author: Hyun Hee Kim

Department of Physical Therapy, Shinsung University, 1 Daehak-ro, Jeongmi-myeon, Dangjin 343-861, Republic of Korea

Tel: 82-41-350-1351 Fax: 82-41-350-1355 E-mail: ksuccess71@shinsung.ac.kr

(c) This is an Open-Access article distributed under the terms of the Creative Commons Attribution Non-Commercial License (http://creativecommons.org/licens es/by-nc/4.0) which permits unrestricted non-commercial use, distribution, and reproduction in any medium, provided the original work is properly cited.

Copyright @ 2015 Korean Academy of Physical Therapy Rehabilitation Science 
strength was based on the improvement of muscle activation of quadriceps, which was caused by the changes in learning effects and pattern of motion units of quadriceps.

Stretching is an exercise that improves the range of joint motion by increasing the extensibility of muscle-tendon units [5]. Basmajian et al. [6] reported that a contracted hamstring caused restrictions on stretching of knee joints, so it increased the pressure on patellofemoral joint during the stance phase of walking. In the same manner, the stiffness in gastrocnemius and soleus muscle causes a compensative pronation at subtalar joint, which results in the increase of internal rotation in femur. Doucette and Goble [7] encouraged patellofemoral pain syndrome (PFPS) patients to take a stretching exercise for iliotibial band, and identified that the flexibility had increased, but the pain had been reduced. It seems that a rehabilitation program for PFPS patients shall include a stretching exercise because the vastus lateralis, gluteal muscle, hamstring, gastrocnemius and flexors of hip joint may contribute to the contraction.

It is said that a meditation may have an effect on and bring about epoch-making changes in prevention and treatment of psychogenic diseases through gathering up shattered nerves, intercepting unnecessary information, regaining the harmony of body and mind, and maintaining physiological stability [8].

The characteristics of human body motion can be measured by using an electromyography (EMG) [9], and the temporal or frequency nature may be used in evaluating the function of muscles and nervous system [10] .

When the taping treatment is applied to a patient with PFPS during stair ascent, the onset timing of EMG of vastus medialis was initiated more fast, and the onset timing of EMG of vastus lateralis was relatively delayed [11]. In the case of strengthening exercise for quadriceps, adduction position of hip joint shortened the onset timing of EMG of vastus medialis more than the onset timing of EMG of vastus lateralis [12].

Jumping is the basic motion that is important in leaping higher, faster and further when basketball and volleyball players, or athletes are trained. And the muscles used in jumping motion are gluteus maximus, quadriceps, and gastrocnemius. This motion training helps increase the power by improving the muscle strength and reaction time of the muscle in operation [13]. Hay [14] suggested that long legs are advantageous in jumping exercise, and athletes should have muscle strength and quick adaptation. Bedi et al. [15] argued that the training focused on strengthening the knee and hip joint muscles have positive effects on jumping ability. Yoo [16] reported that plyometric and weight training had a significant impact on improvement of each muscle, including the jumping ability.

Therefore, this study aims to investigate the EMG onset timing of muscle activation of rectus femoris and gastrocnemius muscle to identify the effects of strengthening and stretching exercises during a jump.

\section{Methods}

\section{Subjects}

The participants in this study consist of a total of 10 college students from Shinsung University, who were healthy 5 male and 5 female students enrolled in the school. The average age of male participants was 23.8 years old, and their average height and weight were $170.6 \mathrm{~cm}$ and $60.6 \mathrm{~kg}$, respectively (Table 1). The participants had no problems in moving their knees due to a degenerative joint disease, musculotendinous injury, or PFPS.

\section{Outcome measure}

The 8-channel surface EMG System (MyoSystem 1400A; Noraxon Inc., Scottsdale, AZ, USA) was used to measure the muscular activities. And the MyoResearch XP master edition 1.06 program (Noraxon Inc.) was used to store and process the signals of EMG.

\section{Procedures}

The subjects wearing casual clothes were asked to stand looking straight ahead so that the EMG onset timing of rectus femoris and gastrocnemius could be measured when the subject was vertical jumping. The subjects were trained to vertical jump when they heard the beeper sound, and they were asked to vertical jump three times according to the sound. After carrying out a pre-test, muscle strengthening, stretching exercises, and a meditation, were carried out. And then, in order to exclude the effects based on order, the participants were asked to pick draw a card with an intervention

Table 1. General characteristics of the subjects $\quad(\mathrm{N}=10)$

\begin{tabular}{lrrr}
\hline Characteristic & Male $(\mathrm{n}=5)$ & \multicolumn{1}{c}{ Female $(\mathrm{n}=5)$} & \multicolumn{1}{c}{ Total } \\
\hline Age $(\mathrm{y})$ & $24.8(1.30)$ & $22.8(0.45)$ & $23.8(1.40)$ \\
Height $(\mathrm{cm})$ & $177.4(1.95)$ & $163.8(3.35)$ & $170.6(7.62)$ \\
Weight $(\mathrm{kg})$ & $69.2(4.92)$ & $52.0(3.39)$ & $60.6(9.90)$ \\
\hline
\end{tabular}

Values are presented as mean (SD). 
method on it, and the experiment was performed according to the order.

As for the strengthening exercise for rectus femoris, the subjects were asked to do an isometric contraction exercise causing a maximum resistance at their ankle joint three times per 10 seconds, which was repeated 5 times, when they were straightening their knees in a sitting position on a table (Figure 1).

As for the muscle strengthening exercise for gastrocnemius, the subjects were asked to lift their heels three times per 10 seconds, which was repeated 5 times, as they were standing upright (Figure 2).

As for the muscle stretching exercise for rectus femoris, the subjects were asked to lie face down and bend their knees as much as possible 5 times in 30 minutes (Figure 3).

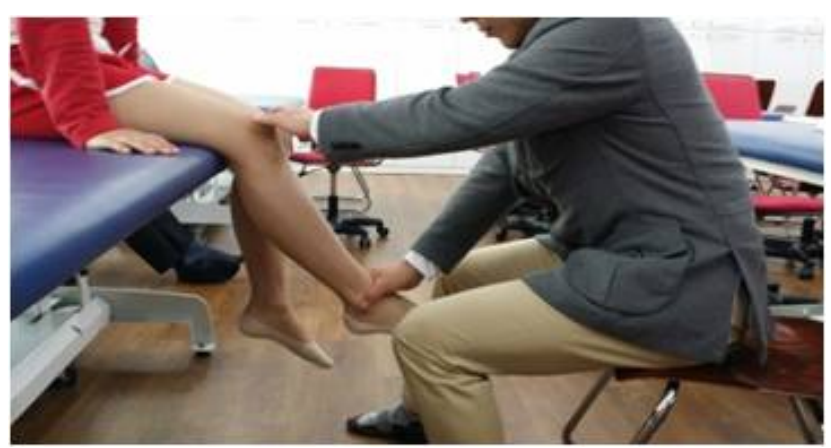

Figure 1. Muscle strengthening exercise for rectus femoris.

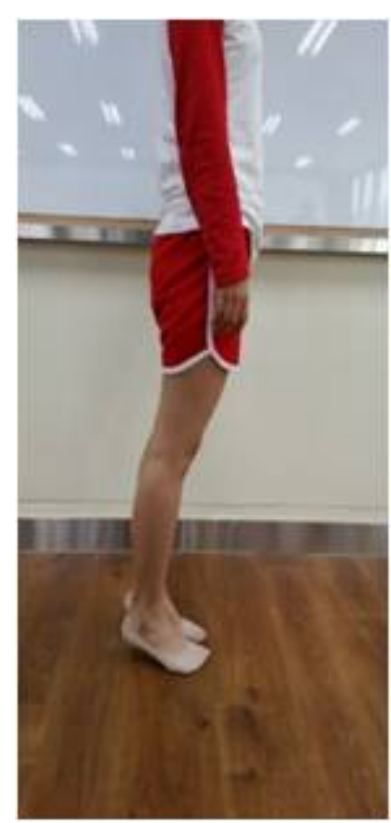

Figure 2. Muscle strengthening exercise for gastrocnemius.
As for the muscle stretching exercise for gastrocnemius, the subjects were asked to maintain ankle joint dorsiflexion for 5 minutes as they were standing upright by using a $\mathrm{Q}$ board (Figure 4).

And also, the meditation was performed to imagine the jump motion in accordance with the beeper sound for five minutes, listening to a quiet music in a comfortable sitting position.

After going through each intervention process, the subjects were asked to jump three times when beeper sounded in the same way as the pre-test, and the average value of each test was used in the analysis.

The sampling rate of EMG signal was set at $1,000 \mathrm{~Hz}$, and the collected data was processed by using a $20-500 \mathrm{~Hz}$ band-pass filter and $50 \mathrm{~Hz}$ low-pass filter after the full wave

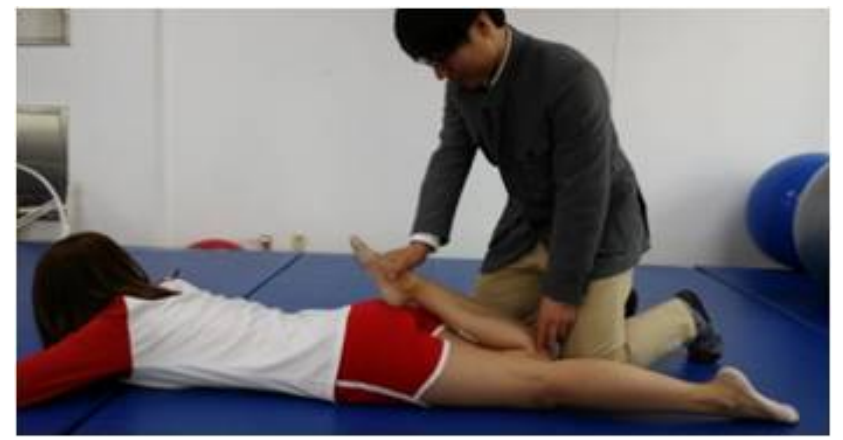

Figure 3. Muscle stretching exercise for rectus femoris.

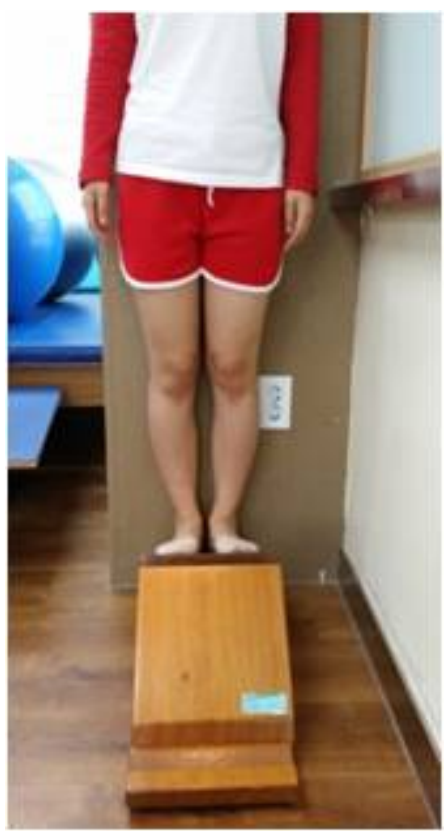

Figure 4. Muscle stretching exercise for gastrocnemius. 
was rectified. The onset timingwas determined as the point at which the EMG signal deviated by more than three standard deviations for at least $25 \mathrm{~ms}$ above the baseline level. The baseline level was based on the averaging of $200 \mathrm{~ms}$ of EMG activity before the commencement of vertical jumping [17]. The electrode was attached on the rectus femoris in the middle between the center of patella and anterior superior iliac spine, and the other electrode attached on the gastrocnemius, which was a protruding part that was $2 \mathrm{~cm}$ away from the middle of calf to the edge [18]. The electrodes (T246H; Bioprotech, Wonju, Korea) used in the experiment were adhesive $\mathrm{Ag} / \mathrm{AgCl}$ surfaces that included a low-allergenic gel.Thediameter of the conductive area was $1 \mathrm{~cm}$; the interelectrode distance was $2 \mathrm{~cm}$ (Figure 5).

\section{Data analysis}

In order to confirm the EMG onset timing of rectus femoris and gastrocnemius according to the internvention, the one-way repeated ANOVA was carried out. When sig-

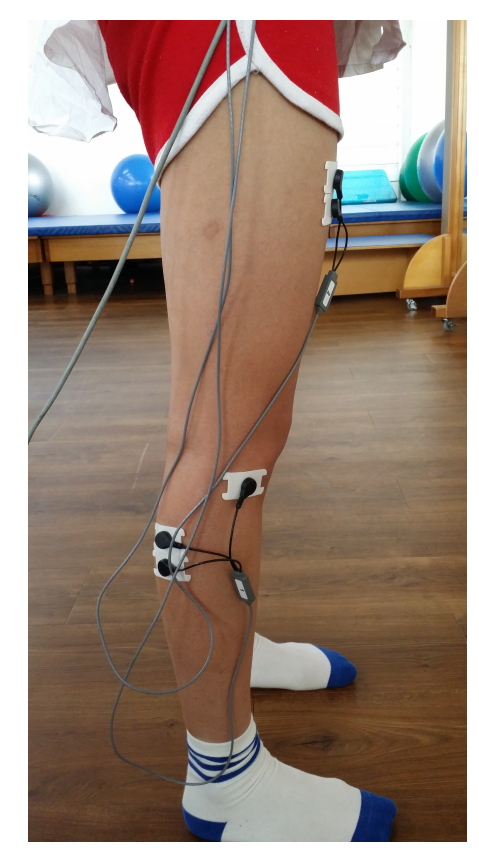

Figure 5. Electrode placement. nificance was confirmed, the Bonferroni method was applied to make a multiple comparison as a post hoc analysis. The significance level was set at 0.05 , and the GreenhouseGeisser correction was performed if the assumption of sphericity was violated. The collected data was analyzed by using the window SPSS ver. 12.0 (SPSS Inc., Chicago, IL, USA), which was a commercial statistical program.

\section{Results}

There was a significant difference in the EMG onset timing of gastrocnemius, while there was no significant difference in the EMG onset timing of rectus femoris according to intervention. After carrying out muscle strengthening exercise, the EMG onset timing of gastrocnemius was 527.17 (113.87) ms, which was significantly faster than EMG onset timing after meditation, which was 559.20 (55.90) ms (Table 2).

\section{Discussion}

The jump motion that enables athletes to move faster, higher and further in a brief moment is essential for improvement of athletic performance. Various training methods have been suggested in order to improve the quick adaptation. In this study, the muscle strengthening and stretching exercise focused on rectus femoris and gastrocnemius, which play a major role in jumping, and the image building training were carried out, so that the EMG onset timing of rectus femoris and gastrocnemius has been identified, which may have an impact on the quick adaptation when jumping.

Park [19] reported that there was no significant difference in reaction time of jump motion after completion of 6-week plyometric and isokinetic training. Callaghan and Oldham [20] confirmed the degree of muscle activation in rectus femoris was improved by taking an isometric training focused on rectus femoris for three months, during which the subjects carried out the training program composed of four 15-minute exercise sets a day.

Table 2. Onset timing of rectus femoris and gastrocnemius according to intervention

\begin{tabular}{cllllll}
\hline \multicolumn{1}{c}{ Variable } & Pre-intervention & Strengthening & Stretching & Meditation & $F$ & $p$ \\
\hline Rectus femoris (ms) & $482.80(79.71)$ & $414.85(78.99)$ & $520.02(85.70)$ & $514.50(88.76)$ & 1.855 & 0.161 \\
Gastrocnemius (ms) & $592.33(101.72)$ & $527.17(113.87)^{\mathrm{a}}$ & $581.37(44.46)$ & $559.20(55.90)$ & 4.726 & 0.009 \\
\hline
\end{tabular}

Values are presented as mean (SD).

${ }^{a}$ Significant difference between strengthening and meditation $(p<0.05)$. 
Cowan et al. [21] reported that when the knee bone taping was applied to a patient with PFPS and the patient was walking up the steps, the EMG onset timing of vastus medialis and vastus lateralis brought about a temporary change, but placebo taping could not make a change. And there was no change in the EMG onset timing of vastus medialis and vastus lateralis when applying the knee bone taping to a normal person. Karst and Willett [22] applied various knee joint exercise to normal persons, but they reported that the EMG onset timing of vastus medialis and vastus lateralis did not vary according to the kinds of exercises. According to the results of this study, the EMG onset timing of rectus femoris appeared not to have any significant difference according to the intervention, but the EMG onset timing of gastrocnemius became faster after carrying out muscle strengthening exercise in comparison with meditation. So, it is assumed that the muscle strengthening exercise has an impact on the quick adaptation of rectus femoris, and the difference from the meditation is that the stable state of body, which is regarded as the characteristics of meditation does not have a positive effect on the jump motion that requires a quick adaptation.

It was reported that a stretching exercise for gastrocnemius, which was taken in advance, reduced the height of jump by $5.6 \%$ [23]. In this study, it was not possible to confirm the statistical significance regarding the EMG onset timing of rectus femoris and gastrocnemius after a stretching exercise, however, it was found out that the EMG onset timing of rectus femoris was delayed after a stretching exercise in comparison with the EMG onset timing measured before intervention. And the EMG onset timing of gastrocnemius increased unlike the study results reported by Wallmann et al. [23]. However, the statistical significance would not be identified. The EMG onset timing of both muscle was delayed after a stretching exercise rather than a muscle strengthening exercise.

In this study, the EMG onset timing has been identified, which has effect on the quick adaptation of rectus femoris and gastrocnemius through a one cross-sectional intervention. And also, it has been found out that a muscle strengthening exercise can advance the EMG onset timing of gastrocnemius. However, it was difficult to compare the results of this study with those of precedent studies as there were not many precedent studies regarding changes in the EMG onset timing. It is important to improve quick adaptation in order to raise exercise capacities. However, it is not easy to change the EMG onset timing, which is the temporal characteristics of muscle, through carrying out the intervention once, like this study. So, it is expected that the effects of long-term exercise on quick adaptation will be identified in the future studies.

In this study, the muscle strengthening and stretching exercise for rectus femoris and gastrocnemius, including the image building training, have been carried out in order to improve the quick adaptation in jump performance, which is closely associated with sports activities. The EMG onset timing of rectus femoris and gastrocnemius has been identified according to the intervention, and it has been found out that the EMG onset timing of gastrocnemius can be shortened after a muscle strengthening exercise. Therefore, we may conclude that the muscle strengthening exercise may have a positive effect on the improvement of quick adaptation of gastrocnemius. And also, it is expected that the future studies will identify the effects of such exercises, which has an effect on jump performance improved by a longer intervention period.

\section{Conflict of Interest}

The authors declared no potential conflicts of interest with respect to the authorship and/or publication of this article.

\section{References}

1. Komi PV. Physiological and biomechanical correlates of muscle function: effects of muscle structure and stretch-shortening cycle on force and speed. Exerc Sport Sci Rev 1984;12:81-121.

2. Hennessy L, Kilty J. Relationship of the stretch-shortening cycle to sprint performance in trained female athletes. J Strength Cond Res 2001;15:326-31.

3. Jones DA, Rutherford OM. Human muscle strength training: the effects of three different regimens and the nature of the resultant changes. J Physiol 1987;391:1-11.

4. Narici MV, Hoppeler H, Kayser B, Landoni L, Claassen H, Gavardi C, et al. Human quadriceps cross-sectional area, torque and neural activation during 6 months strength training. Acta Physiol Scand 1996;157:175-86.

5. Arnheim DD, Prentice WE. Principles of athletic training. 8th ed. St. Louise: Mosby College Publishing; 1993.

6. Basmajian JV, Gopal DN, Ghista DN. Electrodiagnostic model for motor unit action potential (MUAP) generation. Am J Phys Med 1985;64:279-94.

7. Doucette SA, Goble EM. The effect of exercise on patellar tracking in lateral patellar compression syndrome. Am J Sports Med 1992;20:434-40.

8. Eom KY. A study on the effect of meditation through Tai Chi training. Master thesis. Iksan: Wonkwang University; 2008. 
9. Soderberg GL, Knutson LM. A guide for use and interpretation of kinesiologic electromyographic data. Phys Ther 2000;80:485-98.

10. Carey JR, Allison JD, Mundale MO. Electromyographic study of muscular overflow during precision handgrip. Phys Ther 1983; 63:505-11.

11. Gilleard W, McConnell J, Parsons D. The effect of patellar taping on the onset of vastus medialis obliquus and vastus lateralis muscle activity in persons with patellofemoral pain. Phys Ther 1998;78:25-32.

12. Choi BR, Kim MH, Jeon HS. Comparisons of vastusmedialis and vastuslateralis EMG onset time during quadriceps strengthening exercise in neutral and adducted hip positions. Phys Ther Korea 2009;16:42-9.

13. Chu DA, Plummer L. The language of plyometrics. Natl Strength Cond Assoc J 1984;6:30-1.

14. Hay JG. The biomechanics of sports techniques. 4th ed. Englewood Cliffs, NJ: Prentice-Hall; 1993.

15. Bedi JF, Cresswell AG, Engel TJ, Nicol SM. Increase in jumping height associated with maximal effort vertical depth jumps. Res Q Exerc Sport 1987;58:11-5.

16. Yoo WS. Effects in leg muscular strength training for jumping ability to increase woman's volleyball national athletes. Master thesis. Yongin: Myongji University; 2002.

17. Boling MC, Padua DA, Blackburn JT, Petschauer M, Hirth C.
Hip adduction does not affect VMO EMG amplitude or VMO: VL ratios duringa dynamic squat exercise. J Sport Rehabil 2006;15:195-205.

18. Cram JR, Kasman GS, Holtz J. Introduction to surface electromyography. 1st ed. Gaithersburg: Aspen; 1998.

19. Park HC. Effects of plyometric training and isokinetic training on power, agility, isokinetic muscle strength and jump performance ability improvement. Master thesis. Seoul: Korea University; 2006.

20. Callaghan MJ, Oldham JA. The role of quadriceps exercise in the treatment of patellofemoral pain syndrome. Sports Med 1996; 21:384-91.

21. Cowan SM, Hodges PW, Bennell KL, Crossley KM. Altered vastii recruitment when people with patellofemoral pain syndrome complete a postural task. Arch Phys Med Rehabil 2002; 83:989-95.

22. Karst GM, Willett GM. Onset timing of electromyographic activity in the vastus medialis oblique and vastus lateralis muscles in subjects with and without patellofemoral pain syndrome. Phys Ther 1995;75:813-23.

23. Wallmann HW, Mercer JA, McWhorter JW. Surface electromyographic assessment of the effect of static stretching of the gastrocnemius on vertical jump performance. J Strength Cond Res 2005;19:684-8. 